\title{
The Olympic Experimental Gymnasium Program and its Association with the Prevalence of Cardiovascular Risk Factors in Adolescents: A Cross-Sectional Study
}

\author{
Carlos Scherr, ${ }^{1}$ Lucas Helal, ${ }^{2,4,5}$ Filipe Ferrari, $2,4,6 @$ Luciano Juaçaba Belém, ${ }^{3}$ Leonardo Correa Castro Fabiano, ${ }^{1}$ \\ Ludmila Talita Pinheiro, ${ }^{1}$ Ricardo Stein ${ }^{2,4,6,7,8(\mathbb{1}}$ \\ Fundação Pró-Coração, ${ }^{1}$ Rio de Janeiro, RJ - Brazil \\ Universidade Federal do Rio Grande do Sul, ${ }^{2}$ Porto Alegre, RS - Brazil \\ Instituto Nacional do Coração, ${ }^{3}$ Rio de Janeiro, RJ - Brazil \\ Programa de Pós-Graduação em Cardiologia e Ciências Cardiovasculares - Faculdade de Medicina - Hospital de Clínicas de Porto Alegre - \\ Universidade Federal do Rio Grande do Sul, ${ }^{4}$ Porto Alegre, RS - Brazil \\ Laboratório de Fisiopatologia do Exercício (LaFiEx) do Hospital de Clínicas de Porto Alegre - Universidade Federal do Rio Grande do Sul, ${ }^{5}$ \\ Porto Alegre, RS - Brazil \\ Grupo de Pesquisa em Cardiologia do Exercício (CardioEx) - Hospital de Clínicas de Porto Alegre - Universidade Federal do Rio Grande do Sul, ${ }^{6}$ \\ Porto Alegre, RS - Brazil \\ Divisão de Medicina Interna - Hospital de Clínicas de Porto Alegre, ${ }^{7}$ Porto Alegre, $R S$ - Brazil \\ Vitta Centro de Bem-Estar Físico, ${ }^{8}$ Porto Alegre, RS - Brazil
}

\section{Abstract}

Background: Cardiovascular disease (CVD) is the leading cause of death worldwide. Physical activity (PA) and appropriate diet, if adopted in childhood and adolescence, may reduce the CVD burden in later life. The Olympic Experimental Gymnasium (OEG) project was implemented to increase the PA levels of students by means of regular physical exercise and healthy eating habits.

Objectives: To estimate and compare the prevalence of CVD risk factors in OEG schools versus regular schools (RSch) and to examine associations between the school environment and CVD risk factors.

Methods: In this cross-sectional study with a comparator group, adolescents aged 12-13 years attending three OEG schools $(n=719)$ and three RSch $(n=394)$ were evaluated after one year of the ongoing program to estimate the prevalence of overweight, pre-hypertension/hypertension, altered glycemia, and lipid profile. An $\alpha$ level of 0.05 was set for statistical analysis. Results: RSch students had higher odds to have high blood pressure (OR 1.86, 1.36-2.54) and to be overweight (OR 1.49, 1.13-1.98) than OEG students. Glucose levels were not altered in most cases regardless of school type, and no differences were found in lipid profile. In the sensitivity analysis stratified by gender, girls from RSch were more likely to have high body mass index than boys.

Conclusions: Exposure of adolescents to the OEG policies was positively associated with an important reduction in CVD risk factors, including high blood pressure and overweight. (Arq Bras Cardiol. 2019; 112(6):775-781)

Keywords: Cardiovascular Diseases/mortality; Hypertension; Overweight; Dyslipidemias; Exercise; Life Style; Child; Adolescent; Diet.

\section{Introduction}

The attributable fraction of deaths due to physical inactivity can reach values around five million persons in the world. ${ }^{1}$ Among adolescents, the prevalence of a sedentary lifestyle is also high. The Study of Cardiovascular Risks in Adolescents (ERICA) found a frequency of $54 \%$ of physical inactivity, which was more prevalent in girls. ${ }^{2}$ The same study, when considering approximately 37,000 subjects, found that higher physical activity (PA) levels were independently associated with cardiovascular risk and with sedentary time. Interestingly, PA levels do not appear to change the association between body mass index (BMI) and cardiovascular risk. ${ }^{3}$

Mailing Address: Ricardo Stein -

Rua João Caetano 20, apt 402. Postal Code 90470-260, Porto Alegre, RS - Brazil

E-mail: rstein@cardiol.br

Manuscript received August 23, 2018, revised manuscript October 03, 2018, accepted October 10, 2018

DOI: $10.5935 / a b c .20190067$
Lifestyle patterns related to eating habits and PA practices established in the school environment can have potential consequences in adulthood. There is evidence that atherosclerosis begins in early life, progressing slowly into elderliness. ${ }^{4}$ Scherr et al. ${ }^{5}$ stated that it is important to consider the presence of cardiovascular risk factors in school children and that they may be related to lifestyle behaviors.

Given that young people spend most of their time in school, the role of school programs cannot be underestimated. ${ }^{6}$ In this respect, the most common ways to increase PA through the school system have been based on engagement in physical education classes and extracurricular physical activities. However, those are often underused.?

School-based interventions to increase PA seem to be feasible as a strategy to reduce risk factors. Knox et al. ${ }^{8}$ reported a decrease in cardiovascular risk factors among 115 participants aged 12 years-old after an 18-week intervention in a secondary school. Similarly, the ACORDA project found a reduction of obesity in adolescents after increasing PA levels with and without dietary advice after an 8-month interdisciplinary approach. ${ }^{9}$ 
In 2012, Rio de Janeiro's city government initiated a project integrating academic and sports training: the Olympic Experimental Gymnasium (OEG). ${ }^{10}$ Emphasis was given to sports practice (two hours a day, five times a week), and five healthy meals were provided to students every day. Conversely, in regular schools (RSch), PA practice is limited (once a week) and students have only one meal per day.

In this context, we aimed to examine potential associations between the school environment in OEG schools versus RSch and important cardiovascular disease (CVD) risk factors in adolescents. We hypothesized that the adolescents attending RSch would be more likely to have risk factors than those attending OEG schools.

\section{Methods}

This article was written according to the standards of the Strengthening the Reporting of Observational Studies in Epidemiology (STROBE) Statement for cross-sectional studies. ${ }^{11}$

\section{Study design}

A cross-sectional study with a comparator group was conducted at six public schools in Rio de Janeiro: three OEG schools and three RSch. Data collection was carried out in the schools from 2013 to 2015. Data were always collected in the mornings, on the same day of the week by the same trained investigator. To draw association inferences, we considered the school type (ER or OEG) as the exposure, and important CVD risk factors as the outcome, treated as categorical variables. A total of 1,113 students were included in a 2:1 allocation ratio to exposure (719 attending OEG schools) and non-exposure (394 attending RSch).

\section{Eligibility criteria and participant selection}

Eligible participants were all regularly enrolled sixth- to ninth-grade students attending the same school for at least one year. Students attending OEG schools needed to comply with the OEG policy of engaging in PA with energy expenditure $>5$ metabolic equivalents (METs) five times a week. Attendance at PA sessions was self-reported by the students or their parents.

When selecting participants for the comparator group, efforts were made to match the students as closely as possible to avoid potential confounding factors. Thus, a match for chronological age, sex, and grade was made. In RSch, students spent about one hour weekly in PA.

At the time of data collection, four OEG schools were operating in Rio de Janeiro, but only three of them had been operating for at least one year. Therefore, all of the OEG schools were enrolled with no a priori sample size planning. RSch, selected by convenience sampling, were located in the same geographic region of the OEG schools, to avoid discrepancies especially due to socioeconomic status. Both OEG and RSch were public schools, attended by students from all regions of the city.

\section{Data collection}

Written informed consent was obtained from all students and their parents/guardians prior to inclusion in the study. The first school visit was then scheduled, and we performed the following procedures: capillary blood collection, medical interview, physical examination and blood pressure (BP) measurement. All data were collected following a circuit format, in which students passed by each station where the measurements were performed.

A trained medical staff interviewed the students and their parents/guardians using a structured questionnaire designed specifically for this study, in which data of sex and age, in addition to past medical history and current relevant health information were obtained. The medical staff also evaluated adolescents, including anthropometric assessment, measurement of BP and blood sample collection.

Capillary blood samples were collected after a 12-hour overnight fasting to determine glucose levels, total cholesterol, and triglycerides (Roche AccuTrend Plus Kits ${ }^{\circledR}$ ). Altered glucose, total cholesterol and triglycerides levels were considered as results greater than $99 \mathrm{mg} / \mathrm{dL}, 170 \mathrm{mg} / \mathrm{dL}$ and $130 \mathrm{mg} / \mathrm{dL}$, respectively. Height and weight were measured using a Filizola ${ }^{\circledR}$ scale, with maximum capacity of $150 \mathrm{~kg}$, and a coupled vertical stadiometer of $220 \mathrm{~cm}$. Based on BMI values, individuals were classified as eutrophic, overweight or obese, according to the percentiles of height and age for each individual subject. Office BP was measured three times in sitting position, using a calibrated aneroid sphygmomanometer in millimeters of mercury. Students with systolic and diastolic BP above the 95th percentile for their respective gender, age and height were considered hypertensive, while students with systolic and diastolic BP values between their 90th and 95th percentiles were classified as pre-hypertensive. All participants included in the analysis had participated in the PA program for at least one year. This was planned to minimize the risk of reverse causality. Reference values were based on the Brazilian Pediatric Society standards. ${ }^{12}$

\section{Statistical analyses}

A descriptive analysis was conducted, and data were expressed as mean and standard deviation for baseline characteristics and continuous variables, and as absolute frequencies and percentages for categorical variables. In the inferential analysis, the Shapiro-Wilk test was used to assess the normality of data distribution. Groups were then compared by an independent sample two-tailed Student's $t$ test for continuous variables, and by the $\chi^{2}$ test for categorical variables.

Association of school exposure with health status outcomes was assessed by a binomial logistic regression, and its diagnostic was performed to support the analysis. The health status outcomes regarding altered triglycerides, cholesterol, overweight/obesity (regarded as one variable), and pre-hypertension/hypertension were dichotomized into 'YES' and 'NO' according to the thresholds recommended by the Brazilian Pediatric Society. ${ }^{12}$ Point estimates for the associations were expressed as odds ratio (OR) with their 95\% confidence intervals $(95 \% \mathrm{Cl})$, and all analyses were adjusted for sex and age. An exploratory sensitivity analysis was planned in cases of disagreement between matching variables. 'OEG School' was chosen as the reference group for all analyses. No imputation for potential missing data was planned, and all available data for each analysis were used. All statistical analyses were conducted in the StataSE package v. 14.0. An $\alpha$ level of 0.05 was set for statistical inferences. 


\section{Results}

A total of 1,113 students were screened, included, and evaluated. Of these, 719 were attending OEG schools and 394 were attending RSch. Table 1 shows comparative data between OEG and RSch students as well as the number of students analyzed for each category.

RSch students had a higher BMI than OEG students ( $21.4 \pm 4.4$ vs $\left.20.5 \pm 4.3 \mathrm{~kg} / \mathrm{m}^{2}, \mathrm{p}<0.001\right)$, and overweight was also more prevalent in RSch students $(33.8 \%$ vs $26.7 \%$, $p=0.001)$. The prevalence of high BP was higher in RSch students than in OEG students (28.5\% vs $16.3 \%, p=0.013)$. RSch and OEG schools did not differ in the frequency of altered glycemia, total cholesterol or triglycerides.

Despite our efforts to match the groups for sex, the $\chi^{2}$ test indicated differences between groups, with a higher proportion of girls in RSch than in OEG schools $(64.0 \%$ vs $49.4 \%, p<0.001)$. Due to this difference, we performed a sensitivity analysis stratified by sex and the results are shown in Table 2.

The difference observed in BMI between RSch and OEG students was limited to girls $\left(21.9 \pm 4.5\right.$ vs $20.6 \pm 4.3 \mathrm{~kg} / \mathrm{m}^{2}$, $\mathrm{p}=0.001$ ), with no difference when only boys were compared $\left(20.6 \pm 3.9\right.$ vs $\left.20.3 \pm 4.2 \mathrm{~kg} / \mathrm{m}^{2}, \mathrm{p}=0.564\right)$. There was a difference in the prevalence of overweight between RSch and OEG girls (35.7\% vs $24.3 \%, p=0.002)$, but not among boys $(30.3 \%$ vs $29.0 \%, p=0.777)$. There was no difference in any other parameter assessed.

To provide estimates based on associations, a binary logistic regression model was used for each health marker, adjusted for age and sex, and the results are shown in Table 3.

After adjusting for sex and age, RSch students were more likely to be overweight (OR 1.49, 1.13-1.98) and to have pre-hypertension and hypertension (OR 1.86, 1.36-2.34), while no differences were observed for altered total cholesterol or triglycerides.

After stratifying by sex, due to the previously found gender differences between schools, the girls attending RSch had higher odds to be overweight (OR 1.89, 1.30-2.75) and pre-hypertensive/hypertensive (OR 1.66, 1.10-2.51), with no association with altered total cholesterol and triglycerides.
For boys, only the association between attending RSch and being pre-hypertensive/hypertensive was found to be significant (OR 2.20, 1.37-3.54).

\section{Discussion}

In this cohort study, we confirmed our primary hypothesis that students attending OEG schools had lower cardiovascular risk prevalence than students attending RSch. Our results are important because they highlight the association between a healthy school policy and reduction of CVD risk factors in adolescent students.

A proportion-difference test showed that overweight and pre-hypertension/hypertension were less frequent in OEG schools. The prevalence of overweight students in OEG schools was approximately $11 \%$ lower. The difference was even lower for pre-hypertension/hypertension (approximately 13\%). Glucose levels were not altered in most cases. Although no differences were found between schools, the lipid profile was altered in almost half of the students.

The 2016 ERICA study, the largest cross-sectional study involving all regions of Brazil to assess the frequency of cardiovascular risk factors in students, also evaluated individuals of the same age as ours (12-14 years old) and of the same geographic region (southeast). ${ }^{13,14}$ It should be noted that the ERICA study found a lower rate of pre-hypertension/ hypertension $(6.5 \%, 5.5-7.8 \%)$ and a lower overall prevalence of overweight $(18.4 \%, 15.5-21.6 \%)$ in the subgroup of individuals of the same age range and residents of the same geographic region than those observed in our study. Conversely, Cureau et al. ${ }^{14}$ reported a prevalence of $31.3 \%$ for high BP and $23.9 \%$ for overweight in a similar group. Several reasons may account for these differences, mostly regarding the criteria used to define pre-hypertension/hypertension and overweight, in addition to the use of different methodology, including the measurements obtained by aneroid and oscillometric devices. ${ }^{15}$ As for the differences between OEG schools and RSch, Cureau et al. ${ }^{14}$ provide useful findings to explain them, since the prevalence of physical inactivity in their cohort was estimated around $51 \%$.

Considering the attributable risk fraction of physical inactivity to develop hypertension and obesity, ${ }^{16-18}$ it seems

Table 1 - Comparison of demographic characteristics, anthropometric data and cardiovascular risk factors between students from regular schools (RSch) and Olympic Experimental Gymnasiums (OEG)

\begin{tabular}{lccc}
\hline Variable & OEG & RSch & p value \\
\hline Age $($ years $)$ & $12.6 \pm 1.2(\mathrm{n}=719)$ & $13.3 \pm 1.5(\mathrm{n}=394)$ \\
$\mathrm{BMl}\left(\mathrm{kg} / \mathrm{m}^{2}\right)$ & $20.5 \pm 4.3(\mathrm{n}=716)$ & $21.4 \pm 4.4(\mathrm{n}=394)$ \\
Overweight & $191 / 716(26.7 \%)$ & $133 / 394(33.8 \%)$ & 0.001 \\
Altered glycemia & $1 / 700(0.1 \%)$ & $0 / 393(0.0 \%)$ & $152 / 393(38.7 \%)$ \\
Altered total cholesterol & $270 / 714(37.8 \%)$ & $253 / 387(65.4 \%)$ & 0.01 \\
Altered triglycerides & $403 / 624(64.6 \%)$ & $112 / 393(28.5 \%)$ \\
Pre-hypertension/hypertension & $116 / 712(16.3 \%)$ & 0.80 \\
\hline
\end{tabular}

Data are reported as mean \pm standard deviation or absolute number (percentage). BMI: body mass index; NA: not applicable; p-value obtained by chi-square test or Student's t test. 
Table 2 - Comparison of demographic characteristics, anthropometric data and cardiovascular risk factors between students from RSch and OEG schools, stratified by sex

\begin{tabular}{|c|c|c|c|c|c|c|}
\hline \multirow{2}{*}{ Variable } & \multicolumn{3}{|c|}{ Girls } & \multicolumn{3}{|c|}{ Boys } \\
\hline & OEG & RSch & $p$ value & OEG & RSch & $p$ value \\
\hline Students & $354(49.4 \%)$ & $252(64.0 \%)$ & $<0.001$ & $362(50.6 \%)$ & $142(36.0 \%)$ & $<0.001$ \\
\hline Age (years) & $12.6 \pm 1.2(n=354)$ & $13.4 \pm 1.4(n=252)$ & $<0.001$ & $12.6 \pm 1.1(n=362)$ & $13.2 \pm 1.6(n=142)$ & $<0.001$ \\
\hline $\mathrm{BMI}\left(\mathrm{kg} / \mathrm{m}^{2}\right)$ & $20.6 \pm 4.3(n=354)$ & $21.9 \pm 4.5(n=252)$ & 0.001 & $20.3 \pm 4.2(n=362)$ & $20.6 \pm 3.9(n=142)$ & 0.56 \\
\hline Overweight & $86 / 354(24.3 \%)$ & $90 / 252(35.7 \%)$ & 0.002 & $105 / 362(29.0 \%)$ & $43 / 142(30.3 \%)$ & 0.78 \\
\hline Altered glycemia & $1 / 348(0.3 \%)$ & $0 / 252(0.0 \%)$ & NA & $0 / 352(0.0 \%)$ & $0 / 141(0.0 \%)$ & NA \\
\hline Altered total cholesterol & $146 / 355(41.1 \%)$ & $107 / 252(42.5 \%)$ & 0.74 & $124 / 359(34.5 \%)$ & $45 / 141(31.9 \%)$ & 0.56 \\
\hline Altered triglycerides & $226 / 321(70.4 \%)$ & $162 / 247(65.6 \%)$ & 0.22 & $177 / 303(58.4 \%)$ & $91 / 140(65.0 \%)$ & 0.19 \\
\hline Pre-hypertension/ hypertension & $61 / 352(17.3 \%)$ & $67 / 251(26.7 \%)$ & 0.006 & $55 / 360(15.3 \%)$ & $45 / 142(31.7 \%)$ & $<0.001$ \\
\hline
\end{tabular}

Table 3 - Binary logistic regression models using cardiovascular risk factors as independent variables, adjusted for age and sex

\begin{tabular}{lcccc}
\hline & Overweight & Pre/Hyp & Altered TC & Altered TGL \\
\hline Overall ${ }^{\mathrm{a} b}$ & $(\mathrm{n}=1010)$ & $(\mathrm{n}=1105)$ & $(\mathrm{n}=1104)$ & $(\mathrm{n}=1010)$ \\
RSch & $1.49[1.13-1.98]^{\S}$ & $1.86[1.36-2.54]^{\S}$ & $1.01[0.77-1.31]$ & $0.88[0.66-1.16]$ \\
Girls $^{\mathrm{a}}$ & $(\mathrm{n}=606)$ & $(\mathrm{n}=603)$ & $(\mathrm{n}=606)$ & $(\mathrm{n}=567)$ \\
RSch & $1.89[1.30-2.75]^{\S}$ & $1.66[1.10-2.51]^{\S}$ & $1.03[0.73-1.45]$ & $0.69[0.48-1.01]$ \\
Boys $^{\mathrm{a}}$ & $(\mathrm{n}=404)$ & $(\mathrm{n}=502)$ & $(\mathrm{n}=498)$ & $(\mathrm{n}=443)$ \\
RSch & $1.09[0.71-1.69]$ & $2.20[1.37-3.54]^{\S}$ & $0.95[0.62-1.46]$ & $1.19[0.78-1.82]$ \\
\hline
\end{tabular}

Data are reported as odds ratio and 95\% confidence interval: OR [95\%: lower - upper limit]. Pre/Hyp: pre-hypertensive/hypertensive; TC: total cholesterol; TGL: triglycerides; RSch: regular schools. ${ }^{a}$ adjusted for age, ${ }^{b}$ adjusted for sex, $\S=p<0.05$.

reasonable to hypothesize that the differences in health status between OEG and RSch students can result from the PA policy implemented in the OEG. The time course for weight changes and $\mathrm{BP}$ control by non-pharmacological intervention, ${ }^{19}$ and the association between school PA policy and CVD risk factors ${ }^{20,21}$ could support our hypothesis. As for lipid profile, the absence of differences was not a surprise, once PA is known to have only a slight effect on lipid content. ${ }^{22}$ However, the high prevalence of dyslipidemia needs to be addressed.

In the whole sample, the prevalence of dyslipidemia was almost two times greater in RSch than OEG (38.1\% vs 64.9\%). In the ERICA study, ${ }^{23}$ the frequency of hypercholesterolemia and hypertriglyceridemia was $20.1 \%$ and $7.8 \%$, respectively, in a sample of 38,069 adolescents aged 12-17 years. A possible explanation for the discrepancy between our data and that of literature is that the age of 12-13 years corresponds to the pubertal spurt period in boys and girls, and hormonal and other biological interactions may influence biological markers, making it difficult to correctly quantify them. ${ }^{24}$

It is also important to point out the association between obesity and hypertension. Bloch et al. ${ }^{12}$ reported a higher prevalence of hypertension in obese adolescents (28.4\%) than in overweight (15.4\%) and eutrophic (6.3\%) adolescents. The fraction of hypertension attributable to obesity was $17.8 \%$, which raises the hypothesis that about one-fifth of hypertensive patients would not have high BP if they were not obese. This seems important and could serve as a basis for decision-makers of the potential benefits of increasing school-based PA interventions, including intermediate outcomes as control of hypertension, a major cause of cardiovascular mortality in later life. ${ }^{25}$

Interestingly, while both boys and girls attending RSch were less physically active and had a higher frequency of pre-hypertension/hypertension, only girls appeared to benefit more from attending OEG schools when the goal was control of body weight. A possible explanation for this finding is that boys are usually more active than girls and more frequently engaged in non-scheduled PA. ${ }^{26}$

Regarding our exploratory binomial logistic regression analysis, after adjusting for age and sex, RSch students had higher odds to be overweight and to have pre-hypertension/ hypertension than OEG students, supporting the rationale and results described above. ${ }^{19}$ Actually, no differences were found for altered glycemia or lipid profile. The results of the International Study of Childhood Obesity, Lifestyle and the Environment (ISCOLE), a large multicenter cross-sectional study, demonstrated a positive association between sedentary behavior and obesity, even in the cluster analysis of 6,000 students from 12 countries $^{27}$ and in the evaluation of those meeting the recommendations for 24-hour movement guidelines. ${ }^{28}$ 
Regarding our sensitivity analysis in the logistic regression model, it is important to note that the magnitude of association between the school policy and being overweight increased for girls (OR: from 1.49 [1.13-1.98] to 1.89 [1.13-2.75]) and decreased for boys (OR: from 1.49 [1.13-1.98] to 1.09 [0.71-1.69]), when compared to that of the fully adjusted model. With respect to the association between the school policy and pre-hypertension/hypertension, the magnitude of association slightly decreased for girls (OR: from 1.86 [1.36-2.54] to 1.66 [1.10-2.51]) and increased for boys (OR: from 1.86 [1.36-2.54] to 2.20 [1.37-3.54]), with no difference in lipid profile. ${ }^{29}$

Despite the imbalance between the sexes, which motivated the sensitivity analysis, there may be a rationale to explain the changes in the magnitude of the point-estimation. Previous findings support that, overall, girls are more likely to be overweight than boys - perhaps because boys are more physically active and less sedentary. ${ }^{30}$ Nevertheless, data from NHANES revealed that the prevalence of risk factors for CVD and metabolic syndrome was higher in boys than in girls, ${ }^{31}$ which is in line with he statement that boys are more likely to be pre-hypertensive/hypertensive than girls. ${ }^{32}$ This apparently counterintuitive fact could be supported by the present experiment. Among girls, 606 were overweight, and 603 were diagnosed with pre- or hypertension. Among boys, 404 were overweight and 502 were pre-hypertensive or hypertensive. These numbers indicate that other risk factors, such as family history, may be associated with elevated BP - since at least 98 boys had this condition despite the absence of overweight. Therefore, the interactions between school environment and sex are not sufficient to explain the differences in both overweight and pre-hypertension/hypertension observed in the fully adjusted model and in the model stratified by sex. However, this analysis supports the fact that sex may explain the prevalence of overweight and pre-hypertension/ hypertension in adolescents regardless of PA status.

\section{Limitations and future directions}

The results of this study need to be interpreted in light of some limitations. First, because of the cross-sectional design, we cannot establish a causal relationship between school environment (i.e., school PA policy) and the development of CVD risk factors. Second, we did not measure the level of PA of the participants to verify whether they met the PA recommendations of the school policy - this is why we chose school, instead of PA level, as the exposure. Third, no adjustment was made for socioeconomic status, parental influence, or dietary intake, which might have some influence on the development of CVD risk factors. Finally, the categorization of some continuous variables for analysis cannot be disregarded as a limitation, even though we used thresholds commonly reported in the literature.

On the other hand, the hypothesis raised by our study needs to be addressed in intervention-based studies, like multicenter, cluster randomized controlled trials. We addressed, with a valuable sample size, the prevalence of some CVD risk factors in the school environment of a middle-income country in a chronological age-homogeneous sample. The use of higher PA doses, like that of the OEG policy, need to be tested and confirmed in future experiments.

\section{Conclusion}

The prevalence of pre-hypertension/hypertension and overweight differed between schools, and students attending OEG had lower proportion and odds of developing CVD risk factors.

\section{Author contributions}

Conception and design of the research and Acquisition of data: Scherr C, Belém LJ, Fabiano LCC, Pinheiro LT; Analysis and interpretation of the data: Scherr C, Helal L, Belém LJ, Fabiano LCC, Pinheiro LT, Stein R; Statistical analysis: Scherr C, Helal L, Belém LJ, Fabiano LCC, Pinheiro LT; Obtaining financing: Scherr C, Stein R; Writing of the manuscript and Critical revision of the manuscript for intellectual content: Scherr C, Ferrari F, Helal L, Stein R.

\section{Potential Conflict of Interest}

No potential conflict of interest relevant to this article was reported.

\section{Sources of Funding}

This study was funded by Fundação Pró-Coração and partially funded by Fundo de Incentivo à Pesquisa e Eventos (FIPE/HCPA).

\section{Study Association}

This study is not associated with any thesis or dissertation work.

\section{Ethics approval and consent to participate}

This study was approved by the Ethics Committee of the Instituto Brasileiro de Cardiologia under the protocol number CAAE 14549513.1.0000.5272. All the procedures in this study were in accordance with the 1975 Helsinki Declaration, updated in 2013. Informed consent was obtained from all participants included in the study.

\section{Erratum}

In original article "The Olympic Experimental Gymnasium Program and its Association with the Prevalence of Cardiovascular Risk Factors in Adolescents: A Cross-Sectional Study", consider Carlos Scherr as the correct form for the name of the author Carlos Scheer. 


\section{References}

1. Lee IM, Shiroma EJ, Lobelo F, Puska P, Blair SN, Katzmarzyk PT. Impact of physical inactivity on the World's Major Non-Communicable Diseases. Lancet. 2012;380(9838):219-29.

2. Cureau FV, da Silva TLN, Bloch KV, Fujimori E, Belfort DR, de Carvalho KMB, et al. ERICA: leisure-time physical inactivity in Brazilian adolescents. Rev Saude Publica. 2016;50(Suppl 1):4s.

3. Cureau FV, Ekelund U, Bloch KV, Schaan BD. Does body mass index modify the association between physical activity and screen time with cardiometabolic risk factors in adolescents? Findings from a country-wide survey. Int J Obes (Lond). 2017;41(4):551-9.

4. World Health Organization.(WHO) Prevention of cardiovascular disease: pocket Guidelines for assessment and management of cardiovascular risk. Geneva, 2007.

5. Scherr C, Magalhaes CK, Malheiros W. Lipid profile analysis in school children. Arq Bras Cardiol. 2007;89(2):65-70, 73-8.

6. Wilkins J, Graham G, Parker S, Westfall S, Fraser R, Tembo M. Time in the arts and physical education and school achievement. J Curriculum Stud. 2003;35(6):721-34.

7. National Center for Education Statistics. Digest of Education Statistics: 2008. Washington, DC: National Center for Education Statistics. 2009.

8. Knox GJ, Baker JS, Davies B, Rees A, Morgan K, Cooper SM, et al. Effects of a novel school-based cross-curricular physical activity intervention on cardiovascular disease risk factors in 11- to 14-year-olds: the activity knowledge circuit. Am J Health Promot. 2012;27(2):75-83.

9. Aires L, Silva G, Martins C, Marques E, Lagoa MJ, Ribeiro JC, et al. Exercise intervention and cardiovascular risk factors in obese children. Comparison between obese youngsters taking part in a physical activity school-based programme with and without individualised diet counselling: the ACORDA project. Ann Hum Biol. 2016;43(3):183-90.

10. Brazil. Experimental Olympic Ginasium - OEG [internet]. 2016. [cited 2017 July 07]. Available from: http://www.ginasioexperimentalolimpico. net/sobre.html.

11. von Elm E, Altman DG, Egger M, Pocock SJ, Gotzsche PC, Vandenbroucke JP. Strengthening the Reporting of Observational Studies in Epidemiology (STROBE) statement: guidelines for reporting observational studies. BMJ. 2007;335(7624):806-8

12. Sociedade Brasileira de Pediatria. Departamento Científico de Nutrologia. Obesidade na infância e adolescência - Manual de orientação. $2^{a}$ ed. São Paulo: SBP; 2012

13. Bloch KV, Klein CH, Szklo M, Kuschnir MCC, Abreu GdA, Barufaldi LA, et al. ERICA: prevalences of hypertension and obesity in Brazilian adolescents. Rev Saude Publica. 2016;50(Suppl 1):9s.

14. Cureau FV, Duarte P, dos Santos DL, Reichert FF. Clustering of risk factors for noncommunicable diseases in Brazilian adolescents: prevalence and correlates. J Phys Act Health. 2014;11(5):942-9.

15. Gonçalves VSS, Galvão TF, de Andrade KRC, Dutra ES, Bertolin MNT, de Carvalho KMB, et al. Prevalence of hypertension among adolescents: systematic review and meta-analysis. Rev Saude Publica. 2016;50:27.

16. Chomistek AK, Chiuve SE, Eliassen AH, Mukamal KJ, Willett WC, Rimm EB. Healthy lifestyle in the primordial prevention of cardiovascular disease among young women. J Am Coll Cardiol. 2015;65(1):43-51.
17. Ekelund U, Ward HA, Norat T, Luan J, May AM, Weiderpass E, et al. Physical activity and all-cause mortality across levels of overall and abdominal adiposity in European men and women: the European Prospective Investigation into Cancer and Nutrition Study (EPIC). Am J Clin Nutr. 2015;101(3):613-21.

18. Swift DL, Johannsen NM, Lavie CJ, Earnest CP, Church TS. The role of exercise and physical activity in weight loss and maintenance. Prog Cardiovasc Dis. 2014;56(4):441-7.

19. Bleich SN, Vercammen KA, Zatz LY, Frelier JM, Ebbeling CB, Peeters A. Interventions to prevent global childhood overweight and obesity: a systematic review. Lancet Diabetes Endocrinol. 2018;6(4):332-46.

20. Cobayashi F, Oliveira FLC, Escrivão MAM, Daniela S, Taddei JA Obesidade e fatores de risco cardiovascular em adolescentes de escolas públicas. Arq Bras Cardiol. 2010;95(2):200-6.

21. Pazin DC, Rosaneli CF, Olandoski M, Oliveira ERN, Baena CP, Figueredo AS et al. Waist circumference is associated with blood pressure in children with normal body mass index: a cross-sectional analysis of 3,417 school children. Arq Bras Cardiol. 2017;109(6):509-15.

22. Kraus WE, Houmard JA, Duscha BD, Knetzger KJ, Wharton MB, McCartney $J S$, et al. Effects of the amount and intensity of exercise on plasma lipoproteins. N Engl J Med. 2002;347(19):1483-92.

23. Faria JR, Bento VFR, Baena CP, Olandoski M, Gonçalves LG, Abreu GA, et al. ERICA: prevalence of dyslipidemia in Brazilian adolescents. Rev Saude Publica. 2016;50(Suppl 1):10s.

24. Guedes DP, Guedes JERP, Barbosa DS, Oliveira JAd, Stanganelli LCR Fatores de risco cardiovasculares em adolescentes: indicadores biológicos e comportamentais. Arq Bras Cardiol. 2006;86(6):439-50.

25. Chobanian AV, Bakris GL, Black HR, Cushman WC, Green LA, Izzo JL, et al. The Seventh Report of the Joint National Committee on Prevention, Detection, Evaluation, and Treatment of High Blood Pressure: the JNC 7 report. JAMA. 2003; 289(19):2560-72

26. Hallal PC, Knuth AG, Cruz DKA, Mendes MI, Malta DC. Prática de atividade física em adolescentes brasileiros. Cien Saude Colet. 2010;15(supl 2):3035-42.

27. Dumuid D, Olds T, Lewis LK, Martin-Fernandez JA, Barreira T, Broyles S, etal. The adiposity of children is associated with their lifestyle behaviours: a cluster analysis of school-aged children from 12 nations. Pediatr Obes. 2018;13(2):111-9.

28. Roman-Vinas B, ChaputJP, Katzmarzyk PT, Fogelholm M, Lambert EV, Maher C, et al. Proportion of children meeting recommendations for 24-hour movement guidelines and associations with adiposity in a 12-country study. Int J Behav Nutr Phys Act. 2016;13(1):123.

29. Wang Y. Cross-national comparison of childhood obesity: the epidemic and the relationship between obesity and socioeconomic status. Int J Epidemiol. 2001;30(5):1129-36

30. Whitt-Glover MC, Taylor WC, Floyd MF, Yore MM, Yancey AK, Matthews CE. Disparities in physical activity and sedentary behaviors among US children and adolescents: prevalence, correlates, and intervention implications. J Public Health Policy. 2009;30(1):S309-34.

31. Johnson WD, Kroon JJ, Greenway FL, Bouchard C, Ryan D, Katzmarzyk PT Prevalence of risk factors for metabolic syndrome in adolescents: National Health and Nutrition Examination Survey (NHANES), 2001-2006. Arch Pediatr Adolesc Med. 2009:163(4):371-7.

32. Muntner P, He J, Cutler JA, Wildman RP, Whelton PK. Trends in blood pressure among children and adolescents. JAMA. 2004;291(17):2107-13. 
